\title{
Imaging alterations of cardiomyocyte cAMP microdomains in disease
}

\author{
Alexander Froese ${ }^{1}$ and Viacheslav O. Nikolaev ${ }^{2,3 *}$ \\ ${ }^{1}$ Department of Cardiology and Pulmonology, Heart Research Center Göttingen, Georg August University Medical Center, \\ Göttingen, Germany, ${ }^{2}$ German Center for Cardiovascular Research, University Medical Center Hamburg-Eppendorf, \\ Hamburg, Germany, ${ }^{3}$ Institute of Experimental Cardiovascular Research, Hamburg, Germany
}

$3^{\prime}, 5^{\prime}$-cyclic adenosine monophosphate (cAMP) is an important second messenger which regulates heart function by acting in distinct subcellular microdomains. Recent years have provided deeper mechanistic insights into compartmentalized cAMP signaling and its link to cardiac disease. In this mini review, we summarize newest developments in this field achieved by cutting-edge biochemical and biophysical techniques. We further compile the data from different studies into a bigger picture of so far uncovered alterations in cardiomyocyte cAMP microdomains which occur in compensated cardiac hypertrophy and chronic heart failure. Finally, future research directions and translational perspectives are briefly discussed.

Frank Christian

University of Glasgow, UK

Reviewed by:

Philippe Rondard,

Centre National de la Recherche

Scientifique, France

Eduard Stefan,

University of Innsbruck, Austria

${ }^{*}$ Correspondence:

Viacheslav O. Nikolaev, Institute of Experimental Cardiovascular Research,

University Medical Center

Hamburg-Eppendorf, Martinistrasse 52,

D-20246 Hamburg, Germany v.nikolaev@uke.de

Specialty section:

This article was submitted to Experimental Pharmacology and Drug

Discovery,

a section of the journal

Frontiers in Pharmacology

Received: 29 May 2015 Accepted: 03 August 2015 Published: 25 August 2015

Citation:

Froese A and Nikolaev VO (2015) Imaging alterations of cardiomyocyte

CAMP microdomains in disease.

Front. Pharmacol. 6:172.

doi: 10.3389/fphar.2015.00172
Keywords: cAMP, microdomain, cardiomyocyte, hypertrophy, heart failure, FRET, biosensor

$3^{\prime}, 5^{\prime}$-cyclic adenosine monophosphate (cAMP) is an important second messenger and critical regulator of cardiac function. Stimulation of cardiac contractility by catecholamines and their receptors, in particular $\beta$-adrenoceptors ( $\beta$-ARs) which are central to the well-established physiological fight-or-flight response, leads to generation of cAMP that acts in distinct subcellular microdomains (Fischmeister et al., 2006; Zaccolo, 2009; Perera and Nikolaev, 2013). Such microdomains are formed around specific scaffolding proteins (i.e., A-kinase anchoring proteins or AKAPs) which create multiprotein signalosomes. They contain local pools of kinases targeted to their substrates, certain subsets of phosphodiesterases (PDEs) which are enzymes responsible for local cAMP degradation, protein phosphatases and other molecules. All of them act together to confer specificity of multiple substrate phosphorylation and therefore plethora of physiological responses engaged by the same second messenger cAMP (Buxton and Brunton, 1983; Mauban et al., 2009; Zaccolo, 2009; Diviani et al., 2011). In this mini review, we highlight most recent developments and latest research on cardiomyocyte cAMP microdomains in healthy and diseased cardiomyocytes.

\section{cAMP Compartmentation in Healthy Cardiomyocytes}

In healthy cells, cAMP microdomains are supposed to provide specificity of A-kinase (PKA) substrate phosphorylation at different functionally relevant locations. In terms of contractility, several cAMP microdomains exist around calcium handling proteins such as L-type calcium channels (LTCCs), ryanodine receptors (RyRs), and phospholamban (PLN) which regulates the activity of the cardiac sarcoplasmic/endoplasmic reticulum (SR) calcium ATPase 2a (SERCA2a; Bers, 2002; Lompre et al., 2010). Each of these microdomains contains at least one specific AKAP and one PDE isoform (see Figure 1A). Each PDE family, e.g., PDE4, is comprised of several subfamilies such as $4 \mathrm{~A}, 4 \mathrm{~B}, 4 \mathrm{D}$ which have slightly different catalytic domain structures. Every subfamily usually has several isoforms (e.g., 4D3, 4D5) each having a unique $\mathrm{N}$-terminal sequence responsible for differential subcellular localization (Conti and Beavo, 2007). Functional LTCCs are localized in 


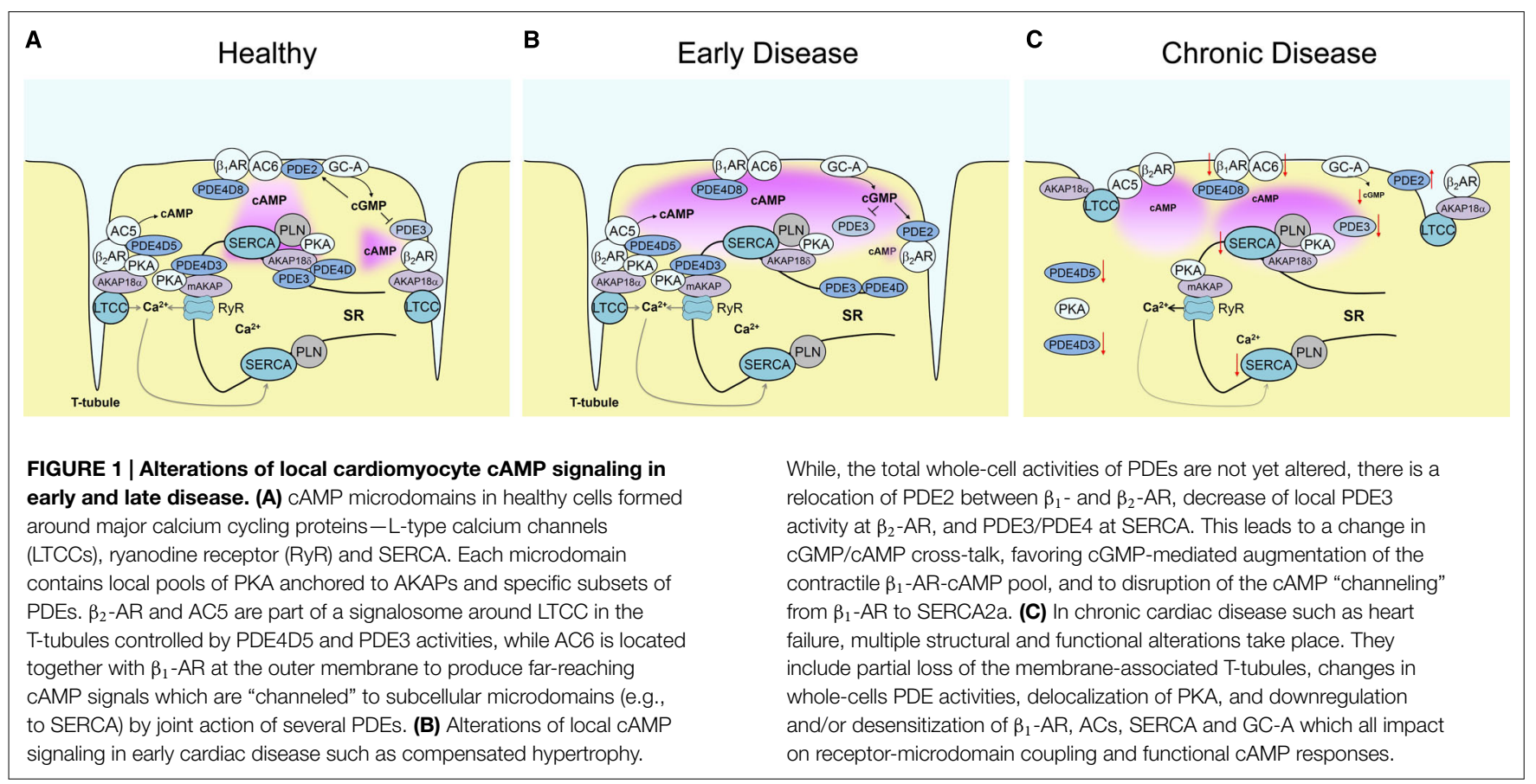

cardiomyocyte transverse (T)-tubules, plasma membrane invaginations rich in caveolin ( $\mathrm{Gu}$ et al., 2002; Insel et al., 2005). Together with AKAP15/AKAP18 $\alpha$ (Fu et al., 2011), AKAP79 (Nichols et al., 2010) and PDE4B (Leroy et al., 2011) they form a signalosome which is crucial for $\beta$-AR/cAMPdependent regulation of LTCC current and contractility. RyRs at the junctional SR have been claimed to be a part of mAKAPorchestrated signalosome which also contains PDE4D3 (Lehnart et al., 2005). PLN forms a complex with AKAP18 $\delta$ (Lygren et al., 2007) and one of the PDE4D and PDE3A isoforms (Beca et al., 2011, 2013) to regulate diastolic calcium uptake. Each of these complexes should also include a local pool of type II PKA molecules. There are two types of regulatory PKA subunits RI and RII, together with catalytic subunits they form either PKA type I or type II complexes. While PKA type II has been shown to phosphorylate the above mentioned calcium handling proteins, the exact nature of PKA type I substrates remains unclear (Stangherlin et al., 2011). Apart from channel recordings and substrate phosphorylation analysis, which provide only indirect measure of the downstream PKA-mediated signaling, it has been challenging to directly visualize cAMP levels in these specific microdomains until novel biophysical techniques became available.

Advent of fluorescence resonance energy transfer (FRET) based biosensors enabled a real-time monitoring of cAMP in intact cells (Sprenger and Nikolaev, 2013). Very early experiments in neonatal cardiomyocytes could directly visualize discrete microdomains where cAMP increases and activates PKA upon $\beta$-AR stimulation (Zaccolo and Pozzan, 2002). There are even different pools of cAMP associated with type I and type II PKA responsible for phosphorylation of different substrates and oppositely regulated by cGMP due to its action on either cGMP-activated PDE2 or cGMP-inhibited PDE3 (Stangherlin et al., 2011). Development of further biosensors, adenoviruses and transgenic mice which serve as a way to express such sensors in cells and tissues, enabled measurements in adult myocytes which revealed tight regulation of cAMP by various PDE families (Warrier et al., 2005; Leroy et al., 2008) and spatial differences between $\beta_{1}$ and $\beta_{2}$-AR-cAMP signals, the former having more diffuse farreaching and the latter highly confined nature (Nikolaev et al., 2006).

Even deeper insights into cAMP compartmentation in relation to membrane structure of living cardiomyocytes were provided by scanning ion conductance microscopy (SICM) combined with FRET. SICM is a non-optical imaging technique which utilizes an electrolyte-filled glass nanopipette as a scanning probe fixed on a three-axis piezo-actuator stage (Korchev et al., 1997). The current flow through pipette is decreased whenever it approaches cell membrane, and by keeping this current change and thereby the distance between pipette tip and cell membrane constant, one can scan the morphological profile of the membrane with nanometer resolution. In general, SICM as a multimodal imaging technique can be applied to study not only cell/tissue structure, but also to record ion channel currents in precise membrane locations, to analyze cell volume and contractility (Miragoli et al., 2011). Using combination of SICM with FRET, which allows local nanopipette-based receptor stimulation and concomitant cAMP imaging, it was uncovered that $\beta_{1}-\mathrm{AR}$ is localized across the whole membrane, while, in contrast, $\beta_{2}-\mathrm{AR}$ is located exclusively in the T-tubules of healthy cells (Nikolaev et al., 2010). Moreover, $\beta_{2} \mathrm{AR}$ are strictly compartmentalized in caveolin3-rich microdomains (Wright et al., 2014) to produce confined cAMP signals limited by local PKA and PDE4 activities. This receptor can also switch from stimulatory to inhibitory G-proteins to limit cAMP production upon prolonged exposure to high agonist concentrations (Liu et al., 2009). 
Not only AKAPs but also cAMP synthetizing enzymes adenylyl cyclases (ACs), with the most predominantly expressed cardiac AC5 and AC6, can center cAMP signalosomes in myocytes and other cells (Cooper and Tabbasum, 2014). A recent elegant electrophysiological study by Timofeyev et al. (2013) using AC5 and AC6 knockout myocytes revealed that AC5 is mainly localized in T-tubules where it interacts with caveolin and $\beta_{2}$ ARs. Together with a local PDE, caveolin is thereby involved in compartmentation of $\beta_{2}$-AR-cAMP signals in this microdomain. In contrast, AC6 associated the $\beta_{1}$-AR is localized outside the Ttubules and is responsible for $\beta_{1}$-AR-mediated augmentation of LTCC current. Interestingly, yet another functional population of $\beta_{1}$-ARs is targeted to T-tubules and the AC5-PDE signalosome (Timofeyev et al., 2013).

To gain more specific insights into cAMP dynamics directly in the microdomains around calcium handling proteins, our group has recently generated targeted FRET biosensors and expressed them in myocardium of transgenic mice to directly monitor local cAMP in freshly isolated adult cardiomyocytes. First, the cAMP sensor Epac1-camps was targeted to caveolinrich membrane microdomains (to generate pmEpac1-camps) where it should localize in close proximity to LTCC and $\beta_{2}$ AR (Perera et al., 2015). This new sensor uncovered differential PDE-dependent regulation of $\beta_{2}$ - and $\beta_{1}$-AR stimulated cAMP pools at the membrane, the former one predominantly confined by PDE3 and the latter one by balanced actions of PDE4, 3, and 2 (Perera et al., 2015). Second, a fusion of Epac1camps with PLN was used to target the cAMP sensor to the SERCA2a microdomain (Sprenger et al., 2015). Here, high basal PDE3 and PDE4 effects were detected which confine this microdomain and prevent PLN phosphorylation by high cytosolic cAMP levels. More interestingly, upon $\beta$-adrenergic stimulation, local, and cytosolic PDE3 and PDE4 act in concert to "channel" cAMP from the membrane to SERCA2a and enable functional response in this microdomain (Sprenger et al., 2015; Figure 1A), a phenomenon which has previously been observed in HEK293 cells when measuring membrane, cytosolic and nuclear cAMP pools (Terrin et al., 2006). However, particularly exiting findings could be made when subjecting both FRET sensor transgenic mouse lines to an experimental model of cardiac disease.

\section{Remodeling of cAMP Microdomains in Early Cardiac Disease}

While alterations in cAMP pathway have been extensively studied in chronic disease (see below), not much is known about changes in local cAMP signaling in early compensated cardiac hypertrophy. To address this question, pmEpacl-camps and Epac1-camps-PLN mice were subjected to transverse aortic constriction which induces only a mild compensated phenotype in the FVB/N1 mouse background. Interestingly, in this case, no changes in total whole-cell PDE activities and no $\beta_{1}$-AR or guanylyl cyclase A (GC-A, membrane receptor which produces cGMP upon natriuretic peptide stimulation) desensitization can be detected. Instead, there was a subcellular relocation of PDE2 between $\beta_{1}$ - and $\beta_{2}-\mathrm{AR}$, and local decrease of the major
PDE3-mediated control at the $\beta_{2}-\mathrm{AR}$ (Figure 1B). This leads to a change of cGMP/cAMP cross-talk in a way that cGMP, which is produced by GC-A stimulated with increased levels of natriuretic peptides in hypertrophy, leads to augmentation of the far-reaching $\beta_{1}$-AR-cAMP pools coupled to increase in force and frequency of contraction (Perera et al., 2015). This might represent a compensatory mechanism aimed to initially maintain cardiac output under the conditions of increased pressure overload during disease, before the transition to a decompensation at some later time-point. However, the exact local mechanisms which accompany this transition remain to be defined. It is also not clear in which particular membrane microdomains GC-A is localized and whether this localization is changed in disease.

The study using cAMP biosensor targeted to SERCA2a demonstrated that cardiac hypertrophy leads to local decrease of PDE3 and PDE4 effects which confine this microdomain from the bulk cytosol. Furthermore, it causes changes in PDE composition at various subcellular locations in a way that leads to impairment of the above described PDE3/4-dependent "channeling" of cAMP from $\beta_{1}$-AR to SERCA2a (Figure 1B, Sprenger et al., 2015). In the future, it would be exciting to dissect which individual PDE3 and PDE4 isoforms are involved in any individual microdomain, how they are regulated by calcium signaling and positive or negative feedback loops, and how all these processes are affected by cardiac disease. This can be done using PDE knockout mouse models, as previously demonstrated for healthy neonatal mouse myocytes using pmEpac-camps and its parential cytosolic sensor (Leroy et al., 2011; Mika et al., 2015). Future developments should also provide new biosensors for other microdomains, such as the one associated with RyR, various other signalosomes and organelles.

\section{cAMP Microdomain Alteration in Chronic Cardiac Disease}

In human and rodent failing myocytes, a series of well-established signaling alterations occurs, including desensitization/downregulation of $\beta_{1}-\mathrm{AR}, \mathrm{GC}-\mathrm{A}, \mathrm{ACs}$, SERCA2a, and impairment of PKA-dependent phosphorylation of major contractile substrates (Lohse et al., 2003). Structurally, SICM studies in failing human and rat cardiomyocytes revealed a loss of cell-surface T-tubules as well as disruption of Z-groove structure (Lyon et al., 2009). The whole-cell activities of major PDE families were reported to be down- (for PDE3/4; Ding et al., 2005; Abi-Gerges et al., 2009) or upregulated (PDE2; Mehel et al., 2013). Loss of membrane T-tubules leads to redistribution of $\beta_{2}$-AR to detubulated areas where it gets uncoupled from its microdomain and generates far-reaching cAMP signals (Nikolaev et al., 2010; see Figure 1C). Altered cAMP compartmentation worsens PKA substrate phosphorylation and calcium cycling. Several open question still remain. Is there any PDE relocation also in chronic disease? Are there any differences between various clinical types of heart failure and what is the time course of deleterious events (detubulation, receptor relocation, microdomain remodeling) during progression of disease? Still unclear are the exact molecular mechanisms behind the loss of T-tubules and their link to calcium cycling, improvement of which correlates with restoration of the membrane structure (Lyon et al., 2012). 
Better understanding of cAMP dynamics in various functionally relevant microdomains and especially of their changes in disease should ultimately provide more precise ways of therapeutic correction. To improve cAMP or cGMP flow in the microdomains, one can imagine approaches aimed at depletion of specific PDEs or PKA from desired signalosomes. More specific PDE inhibition and treatments aimed at improvement of membrane structure, receptor localization and protein composition of the microdomains can also be considered. These developments should enable more targeted and specific cardiac therapeutics.

\section{References}

Abi-Gerges, A., Richter, W., Lefebvre, F., Mateo, P., Varin, A., Heymes, C., et al. (2009). Decreased expression and activity of cAMP phosphodiesterases in cardiac hypertrophy and its impact on ( $\beta$-adrenergic cAMP signals. Circ. Res. 105, 784-792. doi: 10.1161/CIRCRESAHA.109.197947

Beca, S., Ahmad, F., Shen, W., Liu, J., Makary, S., Polidovitch, N., et al. (2013). Phosphodiesterase type 3A regulates basal myocardial contractility through interacting with sarcoplasmic reticulum calcium ATPase type 2a signaling complexes in mouse heart. Circ. Res. 112, 289-297. doi: 10.1161/CIRCRESAHA.111.300003

Beca, S., Helli, P. B., Simpson, J. A., Zhao, D., Farman, G. P., Jones, P. P., et al. (2011). Phosphodiesterase $4 \mathrm{D}$ regulates baseline sarcoplasmic reticulum $\mathrm{Ca}^{2+}$ release and cardiac contractility, independently of L-type $\mathrm{Ca}^{2+}$ current. Circ. Res. 109, 1024-1030. doi: 10.1161/CIRCRESAHA.111.250464

Bers, D. M. (2002). Cardiac excitation-contraction coupling. Nature 415, 198-205. doi: $10.1038 / 415198$ a

Buxton, I. L., and Brunton, L. L. (1983). Compartments of cyclic AMP and protein kinase in mammalian cardiomyocytes. J. Biol. Chem. 258, 10233-10239.

Conti, M., and Beavo, J. (2007). Biochemistry and physiology of cyclic nucleotide phosphodiesterases: essential components in cyclic nucleotide signaling. Annu. Rev. Biochem. 76, 481-511. doi: 10.1146/annurev.biochem.76.060305.150444

Cooper, D. M., and Tabbasum, V. G. (2014). Adenylate cyclase-centred microdomains. Biochem. J. 462, 199-213. doi: 10.1042/BJ20140560

Ding, B., Abe, J., Wei, H., Huang, Q., Walsh, R. A., Molina, C. A., et al. (2005). Functional role of phosphodiesterase 3 in cardiomyocyte apoptosis: implication in heart failure. Circulation 111, 2469-2476. doi: 10.1161/01.CIR.0000165128.39715.87

Diviani, D., Dodge-Kafka, K. L., Li, J., and Kapiloff, M. S. (2011). A-kinase anchoring proteins: scaffolding proteins in the heart. Am. J. Physiol. Heart Circ. Physiol. 301, H1742-H1753. doi: 10.1152/ajpheart.00569.2011

Fischmeister, R., Castro, L. R., Abi-Gerges, A., Rochais, F., Jurevicius, J., Leroy, J., et al. (2006). Compartmentation of cyclic nucleotide signaling in the heart: the role of cyclic nucleotide phosphodiesterases. Circ. Res. 99, 816-828. doi: 10.1161/01.RES.0000246118.98832.04

Fu, Y., Westenbroek, R. E., Yu, F. H., Clark, J. P. III, Marshall, M. R., Scheuer, T., et al. (2011). Deletion of the distal C terminus of $\mathrm{Ca}_{V} 1.2$ channels leads to loss of $\beta$ adrenergic regulation and heart failure in vivo. J. Biol. Chem. 286, 12617-12626. doi: 10.1074/jbc.M110.175307

Gu, Y., Gorelik, J., Spohr, H. A., Shevchuk, A., Lab, M. J., Harding, S. E., et al. (2002). High-resolution scanning patch-clamp: new insights into cell function. FASEB J. 16, 748-750. doi: 10.1096/fj.01-1024fje

Insel, P. A., Head, B. P., Ostrom, R. S., Patel, H. H., Swaney, J. S., Tang, C. M., et al. (2005). Caveolae and lipid rafts: $G$ protein-coupled receptor signaling microdomains in cardiac myocytes. Ann. N. Y. Acad. Sci. 1047, 166-172. doi: 10.1196/annals.1341.015

Korchev, Y. E., Bashford, C. L., Milovanovic, M., Vodyanoy, I., and Lab, M. J. (1997). Scanning ion conductance microscopy of living cells. Biophys. J. 73, 653-658. doi: 10.1016/S0006-3495(97)78100-1

Lehnart, S. E., Wehrens, X. H., Reiken, S., Warrier, S., Belevych, A. E., Harvey, R. D., et al. (2005). Phosphodiesterase $4 \mathrm{D}$ deficiency in the ryanodinereceptor complex promotes heart failure and arrhythmias. Cell 123, 25-35. doi: 10.1016/j.cell.2005.07.030

\section{Author Contributions}

$\mathrm{AF}$ and $\mathrm{VN}$ discussed the concept, wrote and edited the manuscript.

\section{Acknowledgments}

The work in authors' laboratory is supported by the Deutsche Forschungsgemeinschaft (grants NI 1301/1, SFB 1002 TP A01, FOR 2060, IRTG1816), DZHK and the Gertraud und Heinz RoseStiftung.

Leroy, J., Abi-Gerges, A., Nikolaev, V. O., Richter, W., Lechene, P., Mazet, J. L., et al. (2008). Spatiotemporal dynamics of $\beta$-adrenergic cAMP signals and L-type $\mathrm{Ca}^{2+}$ channel regulation in adult rat ventricular myocytes: role of phosphodiesterases. Circ. Res. 102, 1091-1100. doi: 10.1161/CIRCRESAHA.107.167817

Leroy, J., Richter, W., Mika, D., Castro, L. R., Abi-Gerges, A., Xie, M., et al. (2011). Phosphodiesterase $4 \mathrm{~B}$ in the cardiac L-type $\mathrm{Ca}^{2+}$ channel complex regulates $\mathrm{Ca}^{2+}$ current and protects against ventricular arrhythmias in mice. J. Clin. Invest. 121, 2651-2661. doi: 10.1172/JCI44747

Liu, R., Ramani, B., Soto, D., De Arcangelis, V., and Xiang, Y. (2009). Agonist dosedependent phosphorylation by protein kinase $A$ and $G$ protein-coupled receptor kinase regulates $\beta_{2}$ adrenoceptor coupling to $G_{i}$ proteins in cardiomyocytes. J. Biol. Chem. 284, 32279-3287. doi: 10.1074/jbc.M109.021428

Lohse, M. J., Engelhardt, S., and Eschenhagen, T. (2003). What is the role of $\beta$-adrenergic signaling in heart failure? Circ. Res. 93, 896-906. doi: 10.1161/01.RES.0000102042.83024.CA

Lompre, A. M., Hajjar, R. J., Harding, S. E., Kranias, E. G., Lohse, M. J., and Marks, A. R. (2010). $\mathrm{Ca}^{2+}$ cycling and new therapeutic approaches for heart failure. Circulation 121, 822-830. doi: 10.1161/CIRCULATIONAHA.109. 890954

Lygren, B., Carlson, C. R., Santamaria, K., Lissandron, V., McSorley, T., Litzenberg, J., et al. (2007). AKAP complex regulates $\mathrm{Ca}^{2+}$ re-uptake into heart sarcoplasmic reticulum. EMBO Rep. 8, 1061-1067. doi: 10.1038/sj.embor.7401081

Lyon, A. R., MacLeod, K. T., Zhang, Y., Garcia, E., Kanda, G. K., Lab, M. J., et al. (2009). Loss of T-tubules and other changes to surface topography in ventricular myocytes from failing human and rat heart. Proc. Natl. Acad. Sci. U.S.A. 106, 6854-6859. doi: 10.1073/pnas.0809777106

Lyon, A. R., Nikolaev, V. O., Miragoli, M., Sikkel, M. B., Paur, H., Benard, L., et al. (2012). Plasticity of surface structures and $\beta_{2}$-adrenergic receptor localization in failing ventricular cardiomyocytes during recovery from heart failure. Circ. Heart Fail. 5, 357-365. doi: 10.1161/CIRCHEARTFAILURE.111.964692

Mauban, J. R., O’Donnell, M., Warrier, S., Manni, S., and Bond M. (2009). AKAPscaffolding proteins and regulation of cardiac physiology. Physiology (Bethesda) 24, 78-87. doi: 10.1152/physiol.00041.2008

Mehel, H., Emons, J., Vettel, C., Wittkopper, K., Seppelt, D., Dewenter, M., et al. (2013). Phosphodiesterase-2 is up-regulated in human failing hearts and blunts $\beta$-adrenergic responses in cardiomyocytes. J. Am. Coll. Cardiol. 62, 1596-1606. doi: $10.1016 /$ j.jacc.2013.05.057

Mika, D., Richter, W., and Conti, M. A. (2015). CaMKII/PDE4D negative feedback regulates cAMP signaling. Proc. Natl. Acad. Sci. U.S.A. 112, 2023-2028. doi: 10.1073/pnas.1419992112

Miragoli, M., Moshkov, A., Novak, P., Shevchuk, A., Nikolaev, V. O., El-Hamamsy, I., et al. (2011). Scanning ion conductance microscopy: a convergent highresolution technology for multi-parametric analysis of living cardiovascular cells. J. R. Soc. Interface 8, 913-925. doi: 10.1098/rsif.2010.0597

Nichols, C. B., Rossow, C. F., Navedo, M. F., Westenbroek, R. E., Catterall, W. A. Santana, L. F., et al. (2010). Sympathetic stimulation of adult cardiomyocytes requires association of AKAP5 with a subpopulation of L-type calcium channels. Circ. Res. 107, 747-756. doi: 10.1161/CIRCRESAHA.109.216127

Nikolaev, V. O., Bunemann, M., Schmitteckert, E., Lohse, M. J., and Engelhardt, S. (2006). Cyclic AMP imaging in adult cardiac myocytes reveals far-reaching $\beta_{1}$ adrenergic but locally confined $\beta_{2}$-adrenergic receptor-mediated signaling. Circ. Res. 99, 1084-1091. doi: 10.1161/01.RES.0000250046.69918.d5 
Nikolaev, V. O., Moshkov, A., Lyon, A. R., Miragoli, M., Novak, P., Paur, H., et al. (2010). $\beta_{2}$-adrenergic receptor redistribution in heart failure changes cAMP compartmentation. Science 327, 1653-1657. doi: 10.1126/science.1185988

Perera, R. K., and Nikolaev, V. O. (2013). Compartmentation of cAMP signalling in cardiomyocytes in health and disease. Acta Physiol (Oxf). 207, 650-662. doi: 10.1111/apha.12077

Perera, R. K., Sprenger, J. U., Steinbrecher, J. H., Hubscher, D., Lehnart, S. E., Abesser, M., et al. (2015). Microdomain switch of cGMP-regulated phosphodiesterases leads to ANP-induced augmentation of $\beta$-adrenoceptorstimulated contractility in early cardiac hypertrophy. Circ. Res. 116, 1304-1311. doi: 10.1161/CIRCRESAHA.116.306082

Sprenger, J. U., and Nikolaev, V. O. (2013). Biophysical techniques for detection of cAMP and cGMP in living cells. Int. J. Mol. Sci. 14, 8025-8046. doi: 10.3390/ijms14048025

Sprenger, J. U., Perera, R. K., Steinbrecher, J. H., Lehnart, S. E., Maier, L. S., Hasenfuss, G., et al. (2015). In vivo model with targeted cAMP biosensor reveals changes in receptor-microdomain communication in cardiac disease. Nat. Commun. 6, 6965. doi: 10.1038/ncomms7965

Stangherlin, A., Gesellchen, F., Zoccarato, A., Terrin, A., Fields, L. A., Berrera, M., et al. (2011). cGMP signals modulate cAMP levels in a compartment-specific manner to regulate catecholamine-dependent signaling in cardiac myocytes. Circ. Res. 108, 929-939. doi: 10.1161/CIRCRESAHA.110.230698

Terrin, A., Di Benedetto, G., Pertegato, V., Cheung, Y. F., Baillie, G., Lynch, M. J., et al. (2006). PGE(1) stimulation of HEK293 cells generates multiple contiguous domains with different [cAMP]: role of compartmentalized phosphodiesterases. J. Cell Biol. 175, 441-451. doi: 10.1083/jcb.200605050

Timofeyev, V., Myers, R. E., Kim, H. J., Woltz, R. L., Sirish, P., Heiserman, J. P., et al. (2013). Adenylyl cyclase subtype-specific compartmentalization: differential regulation of L-type $\mathrm{Ca}^{2+}$ current in ventricular myocytes. Circ. Res. 112, 1567-1576. doi: 10.1161/CIRCRESAHA.112.300370

Warrier, S., Belevych, A. E., Ruse, M., Eckert, R. L., Zaccolo, M., Pozzan, T., et al. (2005). $\beta$-adrenergic- and muscarinic receptor-induced changes in cAMP activity in adult cardiac myocytes detected with FRET-based biosensor. Am. J. Physiol. Cell Physiol. 289, C455-C461. doi: 10.1152/ajpcell.00058. 2005

Wright, P. T., Nikolaev, V. O., O’Hara, T., Diakonov, I., Bhargava, A., Tokar, S., et al. (2014). Caveolin-3 regulates compartmentation of cardiomyocyte $\beta_{2}$ adrenergic receptor-mediated cAMP signaling. J. Mol. Cell Cardiol. 67, 38-48. doi: 10.1016/j.yjmcc.2013.12.003

Zaccolo, M. (2009). cAMP signal transduction in the heart: understanding spatial control for the development of novel therapeutic strategies. Br. J. Pharmacol. 158, 50-60. doi: 10.1111/j.1476-5381.2009.00185.x

Zaccolo, M., and Pozzan, T. (2002). Discrete microdomains with high concentration of cAMP in stimulated rat neonatal cardiac myocytes. Science 295, 1711-1715 doi: $10.1126 /$ science. 1069982

Conflict of Interest Statement: The authors declare that the research was conducted in the absence of any commercial or financial relationships that could be construed as a potential conflict of interest.

Copyright (c) 2015 Froese and Nikolaev. This is an open-access article distributed under the terms of the Creative Commons Attribution License (CC BY). The use, distribution or reproduction in other forums is permitted, provided the original author(s) or licensor are credited and that the original publication in this journal is cited, in accordance with accepted academic practice. No use, distribution or reproduction is permitted which does not comply with these terms. 